\title{
Intraoperative acute brain swelling when performing indirect anastomosis in a patient with moyamoya disease -A case report-
}

\author{
Ji-Sung $\mathrm{Nho}^{1}$, Soo-Eun Choi ${ }^{1}$, Jae-Woo $\mathrm{Yi}^{2}$, and Jong-Man Kang ${ }^{2}$ \\ Department of Anesthesiology and Pain Medicine, ${ }^{1}$ Kyung Hee University Hospital, ${ }^{2}$ Kyung Hee University, Hospital at Gangdong, \\ Seoul, Korea
}

\begin{abstract}
A 6-year-old male patient who was suffering from a cold and a transient ischemic attack was scheduled to undergo encephalo-duro-arterio-synangiosis for treating his moyamoya disease. Acute brain edema occurred just after opening the dura mater. Head elevation, reduction of the head rotation and hyperventilation were done. The inhalational agents were discontinued and total intravenous anesthesia was started. The swelling was reduced after intravenously infusing mannitol. An abrupt return from hypocapnia to normocapnea during the induction of general anesthesia was thought to be the cause of the acute brain swelling. In conclusion, correction of hypocapnea needs to be performed gradually during the induction of anesthesia and when performing an operation for treating a patient
\end{abstract} with moyamoya disease. (Korean J Anesthesiol 2010; 59: S191-S193)

Key Words: Brain edema, Cerebral revascularization, Mannitol, Moyamoya disease.

Patients with moyamoya disease are known to show progressive stenosis and occlusion of the terminal portion of the bilateral internal carotid arteries and they have an abnormal vascular network in the vicinity of arterial occlusions.

In our case, acute brain swelling occurred immediately after opening of the dura mater during bypass surgery for moyamoya disease. The collateral vessels in patients with moyamoya disease appear to retain their ability to constrict during hypocapnia, but not to dilate during hypercapnia. This event may be caused by the increased cerebral blood flow in the normal area, and presumably because of the intracerebral steal phenomenon.

\section{Case Report}

A 6-year-old male patient $(20 \mathrm{~kg}, 112 \mathrm{~cm})$ was diagnosed as having moyamoya disease according to the carotid angiography. His symptoms were headache, numbness and motor weakness of the left lower extremity. He also presented with the mild symptoms of a cold. There was no definite infarction on the brain MRI, but an occlusion of the first segment of the right middle cerebral artery (Suzuki grade III) was founded. He was scheduled to undergo encephalo-duro-arterio-synangiosis.

General anesthesia was achieved with intravenous injection of glycopyrrolate $0.1 \mathrm{mg}$, thiopental sodium $100 \mathrm{mg}$ and

Received: February 22, 2010. Revised: 1st, March 11, 2010; 2nd, April 23, 2010. Accepted: May 3, 2010.

Corresponding author: Jong-Man Kang, Ph.D., Department of Anesthesiology, Kyung Hee University, Hospital at Gangdong, 149, Sangil-dong, Gangdong-gu, Seoul 134-090, Korea. Tel: 82-2-440-6193, Fax: 82-2-440-7808, E-mail: kjm@khnmc.or.kr

(c) This is an open-access article distributed under the terms of the Creative Commons Attribution Non-Commercial License (http:// creativecommons.org/licenses/by-nc/3.0/), which permits unrestricted non-commercial use, distribution, and reproduction in any medium, provided the original work is properly cited. 
atracurium $15 \mathrm{mg}$ and it was maintained with isoflurane. Tracheal intubation was performed after the loss of the twitch response. The patient's lungs were mechanically ventilated with a mixture of nitrous oxide $(50 \%)$ in oxygen (fresh gas flow rate $=3 \mathrm{~L} / \mathrm{min}$, tidal volume $=200 \mathrm{ml}$, frequency $=18$, inspiratory:expiratory ratio $=1: 2$ ). The arterial blood pressure was maintained at $120-130 / 60-70 \mathrm{mmHg}$ with monitoring the radial artery pressure. Intraoperative monitoring also included the heart rate, central venous pressure, end-tidal carbon dioxide, peak airway pressure, urine output and temperature.

The patient was in a supine position with the head rotated 80 degrees opposite to the operation site and the head was elevated 10 degrees without skeletal fixation. The $\mathrm{PaCO}_{2}$ level was maintained to be near $40 \mathrm{mmHg}$ and the peak airway pressure was less than $20 \mathrm{cmH}_{2} \mathrm{O}$.

The brain swelling occurred when the dura mater was cut in an H-pattern, so the arachnoid membrane over the cortical sulci was dissected to drain the cerebrospinal fluid. However, the prominent brain swelling remained constant (Fig. 1). Head elevation, reduction of head rotation and hyperventilation were done to reduce the brain swelling. A blood gas analysis was done, and this revealed $\mathrm{pH}$ of $7.43, \mathrm{PaCO}_{2}$ of $32 \mathrm{mmHg}$ and $\mathrm{PaO}_{2}$ of $281 \mathrm{mmHg}$ with $\mathrm{FiO}_{2}$ of 0.5 and $\mathrm{EtCO}_{2}$ of 31 . The isoflurane and nitrous oxide were discontinued and total intravenous anesthesia (propofol 1-4 $\mu \mathrm{g} / \mathrm{ml}$ and remifentanil $1-4 \mathrm{ng} / \mathrm{ml}$ target organ concentration) was started. Then, the heart rate was 128 beats/min, the mean arterial pressure was $74 \mathrm{mmHg}$ and the central venous pressure was $9 \mathrm{mmHg}$. The swelling was reduced 10 minutes after intravenously injecting $70 \mathrm{ml}$ of $20 \%$ mannitol. The operation was restarted. The total operation time was 5 hours. After surgery, the CT scan revealed no intracranial hemorrhages, local cerebral edema or definite midline deviations. The patient was transferred to the intensive care unit

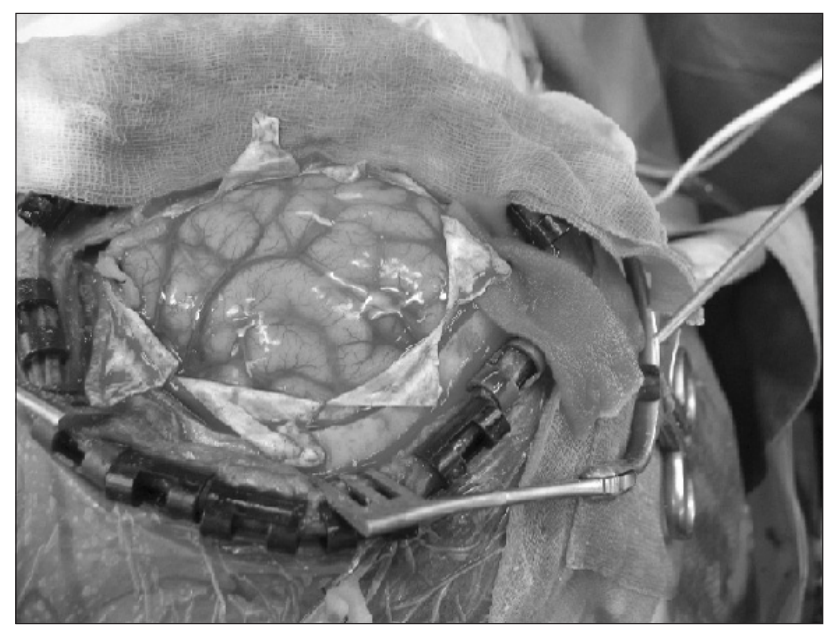

Fig. 1. Intraoperative photograph of the moyamoya case. Acute brain swelling is shown in the operative field. and the lungs were mechanically ventilated. One hour later, the trachea was extubated after spontaneous neuromuscular reversal. One week later, he was discharged without any neurologic deficit.

\section{Discussion}

Patients with moyamoya disease have narrowing or occlusion of the internal carotid arteries and an abnormal vascular network at the base of the brain $[1,2]$. The surgical therapies consist of either direct bypass surgery or indirect bypass procedures such as encephalo-duro-arteriosynangiosis, encephalomyosynangiosis, encephalo-duro-arteriomyosynangiosis and omental transplantation [3].

Brain swelling is thought to be related to cerebral vasodilatation and it is a life-threatening complication in conditions associated with cerebral ischemia [4-6]. The general causes to be excluded are changes in fluid balance, venous stasis, the head position, inhalational agents and an increment in a level of the $\mathrm{PaCO}_{2}$.

In patients with moyamoya disease, the collateral vessels seem to be able to constrict during hypocapnia, but they do not dilate during hypercapnia [7]. It has been asserted that not only hypocapnia, but also hypercapnia decreases the local cortical blood flow as perfused by collateral vessels, and presumably because of an intracerebral steal phenomenon [8]. Touho [9] reported that acute brain swelling occurred in 4 cases of a total of 720 operations for moyamoya disease and Touho described three cases that happened just after opening the dura mater. One of the three cases showed signs of a cold, another manifested allergic rhinitis and the other experienced hyperventilation that resulted in a transient ischemic attack before the operation. The author thought an abrupt return from hypocapnia to normocapnia during the induction of general anesthesia was the cause of the acute brain swelling.

In the present case, there was no prominent increment in the blood pressure before and during the brain swelling. Although the head had been elevated, acute brain swelling occurred anyway. Therefore, it did not seem that the blood pressure and head position caused an increase in the intracranial venous pressure even if there was a possible influence of head rotation on the brain swelling. We also properly maintained the intravascular fluid balance before the event and so overhydration could be ruled out. The effects of inhalational anesthesia on the cerebral circulation in patients who are undergoing revascularization procedures for moyamoya disease were reported to be decreased regional cortical blood flow, increased jugular bulb oxygen saturation and decreased frontal regional oxygen saturation, whereas these effects did not occur with total intravenous anesthesia [10]. Propofol was 
associated with better preservation of the cerebral circulation as compared to that of isoflurane in the case of a child with moyamoya disease during a cerebral angiography procedure [11]. The optimal range of $\mathrm{CO}_{2}$ for isoflurane is more restricted than that for propofol and presumably because isoflurane induces hyperemia by itself and this hyperemia decreases the cerebral blood flow [7]. We changed the inhalational anesthesia to total intravenous anesthesia when the brain swelling started.

Although the exact mechanism for the occurrence of acute intraoperative brain swelling during elective neurosurgery is not known, there are treatment steps that must be taken when it happens. First, it is necessary to check the level of $\mathrm{PaCO}_{2}$ in an awake patient before the anesthetic induction and to adequately maintain the level of $\mathrm{PaCO}_{2}$ during induction of anesthesia. Second, all possible measures, including controlled hyperventilation, hemodynamic management, a suitable position of the head, administration of hypertonic solutions, dexamethasone and possibly barbiturates should be used when acute brain swelling occurs [12]. Finally, in the case of intractable herniation of the brain, a debulking operation such as lobectomy might be considered.

In conclusion, when acute brain swelling occurs, anesthetic as well as the intracranial causes need to be rapidly checked and ruled out as persist brain swelling can lead to irreversible neurological damage to the brain. It is also recommended that correction of hypocapnea needs to be performed gradually during the induction of anesthesia and when operating on a patient with moyamoya disease.

\section{References}

1. Fukui M, Kono S, Sueishi K, Ikezaki K. Moyamoya disease.
Neuropathology 2000; 20 Suppl: S61-4.

2. Peerless SJ. Risk factors of moyamoya disease in Canada and the USA. Clin Neurol Neurosurg 1997; 99 Suppl 2: S45-8.

3. Reis CV, Safavi-Abbasi S, Zabramski JM, Gusmão SN, Spetzler RF, Preul MC. The history of neurosurgical procedures for moyamoya disease. Neurosurg Focus 2006; 20: E7.

4. Whittle IR, Viswanathan R. Acute intraoperative brain herniation during elective neurosurgery: pathophysiology and management considerations. J Neurol Neurosurg Psychiatry 1996; 61: 584-90.

5. Gartshore G, Patterson J, Macrae IM. Influence of ischemia and reperfusion on the course of brain tissue swelling and blood-brain barrier permeability in a rodent model of transient focal cerebral ischemia. Exp Neurol 1997; 147: 353-60.

6. Hwang GS, Rhee KY, Oh YS. Intraoperative contralateral epidural hematoma following removal of cerebral arteriovenous malformation. Korean J Anesthesiol 1994; 27: 1018-25.

7. Oshima H, Katayama Y, Hirayama T. Intracerebral steal phenomenon associated with global hyperemia in moyamoya disease during revascularization surgery. J Neurosurg 2000; 92: 949-54.

8. Yusa T, Yamashiro K. Local cortical cerebral blood flow and response to carbon dioxide during anesthesia in patients with moyamoya disease. J Anesth 1999; 13: 131-5.

9. Touho H. Acute brain swelling during operations for three patients with moyamoya disease resulting in performance of indirect anastomosis as a substitute for direct anastomosis. No Shinkei Geka 2008; 36: 323-7.

10. Sato K, Shirane R, Kato M, Yoshimoto T. Effect of inhalational anesthesia on cerebral circulation in Moyamoya disease. J Neurosurg Anesthesiol 1999; 11: 25-30.

11. Culebras X, Martin JB, Treggiari-Venzi M, Ruefenacht D, Habre W. Propofol increased cerebral perfusion as compared with isoflurane during a cerebral angiography in a child with moyamoya disease. J Neurosurg Anesthesiol 2003; 15: 50-4.

12. Suárez JI. Treatment of acute brain edema. Rev Neurol 2001; 32: 275-81. 